\title{
Optimal design of power supply systems using genetic algorithms
}

\author{
J. R. Jimenez-Octavio \& E. Pilo \\ Instituto de Investigación Tecnológica, \\ E.T.S. de Ingeniería ICAI, Univ. P. Comillas, Madrid, Spain
}

\begin{abstract}
This paper presents an optimization model based on genetic algorithms for designing traction power supply systems. The proposed model is suitable both for planning new lines and for expanding the old ones, resulting in a more efficient operation as well as in lower investment costs. Minimization of fixed installation represents the optimization criterion for searching innovative designs that fulfil certain technical constraints: maximum voltage drops and maximum power consumption in the substations. The variables involved in the optimization problem are: number, type and location of railway overhead lines; and number, size and location of traction substations. Finally, the evaluation of possible designs involves simplified electrical modelling of the studied railway stretch. Thus, electrical simulations and calculations have been also adapted for their implementation in the genetic algorithm. A section of the Madrid-Barcelona high-speed line has been considered as a study case in order to analyze the performance of the proposed model. Results reveal the suitability of the new designs obtained with the presented model and its goodness and robustness.
\end{abstract}

Keywords: railway, power supply system, optimization, genetic algorithms.

\section{Introduction}

The design of the electrification is a complex task that involves different interdisciplinary analysis. Normally, the design process is done iteratively by refining candidate designs based on its estimated performance. In this process simulation tools are crucial to evaluate the performance in many different situations. However, they do not usually include criteria to modify candidate designs in order to obtain the final solution. Thus, the designer has to decide the 
best changes to be done to the candidate design in order to fulfil technical constraints and to reduce costs in a reasonable way. As this is not an obvious task, the development of decision-making computer-aided tools based on optimization models can be very useful to ensure efficient uses of investments.

In [1] simplified models have been formulated to represent bi-voltage $2 \times 25 \mathrm{kV}$ meshed topologies in a simplified way. Based in these models, a optimization procedure has been presented in [2] to select the most efficient centenary and autotransformer combinations. The results of this optimization can be used as an input for the optimization of the power-supply system presented in [3], which formulates a mixed integer programming (MIP) optimization problem. This procedure is suitable for small-medium network sizes (up to 200-250 km), but for bigger problems computation times are too long. In this paper, a genetic algorithm based optimization procedure (GA) is presented and its performance is analyzed. Furthermore, the solutions and computing times are compared to the MIP-based optimization.

The optimization is formulated to minimize the total construction cost of the power supply system while fulfilling technical restrictions. The optimization variables include: (i) substations (number, location), (ii) location of neutral zones, (iii) catenary for every sector (starting and ending point, feeding system, conductors), and (iv) the locations of the required autotransformers. The total construction cost includes: (i) substations, (ii) their connection to the highvoltage network, (iii) catenaries and (iv) autotransformers.

The second section of this paper describes the electrification of AC power supply systems. The third section presents how the genetic algorithm is formulated in order to perform the optimization. In the fourth section, the performance of the proposed method is evaluated in a section of the new MadridBarcelona high-speed line. Finally, the fifth section summarizes the conclusions of this work.

\section{AC power supply}

As shown in Figure 1, the railway electrical system is divided in electricallyisolated single-phased sectors, which are fed from the three-phase network through a traction substation $[4,5]$.

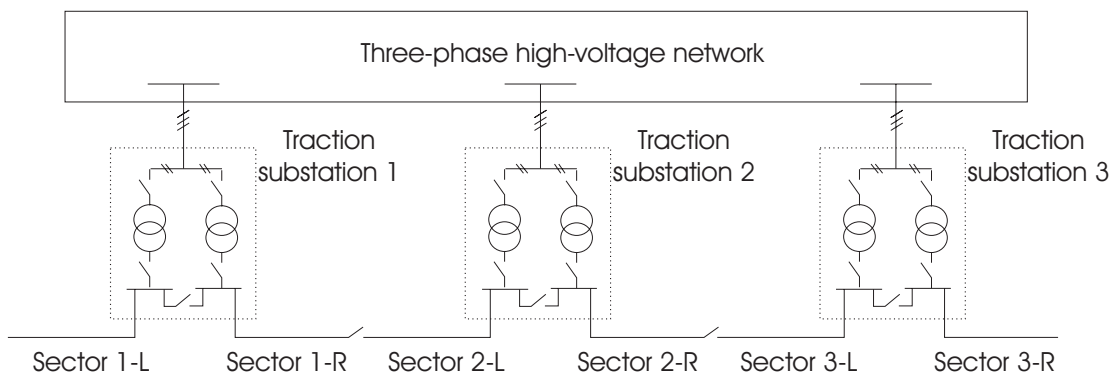

Figure 1: General structure of power supply system. 
These substations are connected between two of the three phases of the highvoltage network. It should be noted that topology could be modified in case of failures to guarantee the operation. For instance, if one of the transformers of a substation fails, the other takes on the corresponding sector.

Each of these sectors can use either mono-voltage system (such as $1 \times 25 \mathrm{kV}$ ) or bi-voltage (such as $2 \times 25 \mathrm{kV}$ ) system. In mono-voltage systems, the feeding conductors are set directly to the specified voltage level (see Figure 2). In bivoltage systems, a higher voltage is set between feeding conductors $[6,7]$. This voltage is reduced by using autotransformers distributed along the catenary (see Figure 3).

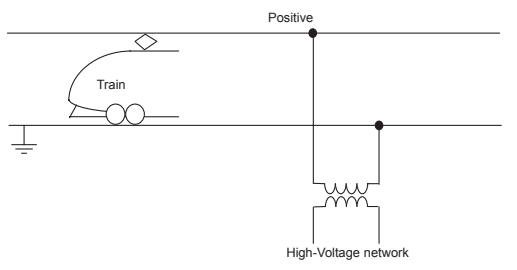

Figure 2: Mono-voltage system configuration.

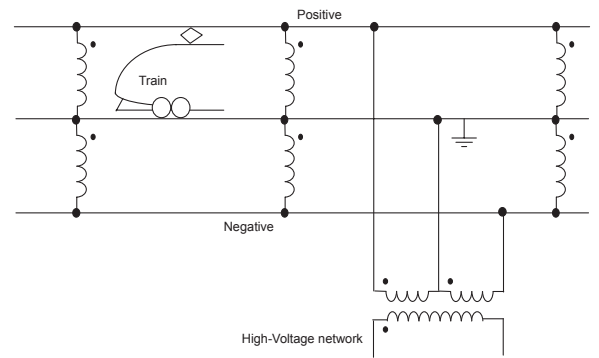

Figure 3: Dual system configuration.

In [1], the behavior of dual systems is analyzed and a model is presented to represent bi-voltage systems as if they were mono-voltage systems. This representation is used in this paper to characterize mono-voltage and bi-voltage systems in a similar manner.

\section{The optimization problem}

An optimization problem formulation has been carried out introducing several criteria for improving classic designs. Particularly, total construction costs are minimized and technical criteria are considered as restrictions. This optimization problem has been tackled using a genetic algorithm.

While solving the optimization problem, the number of sectors (see Figure 2) is determined and thus the number of substations and neutral zones, as well as their location. Moreover, the catenary type (and number of autotransformers in dual systems) of each subsector is obtained. 
The formulation of this problem is organized in five steps. Firstly, the constant parameters are presented. Then, the optimization variables are established. Next, the objective function is defined. The considered restrictions are formulated afterwards and finally, certain parameters and questions related to the genetic algorithm implemented are pointed out.

\subsection{Constant parameters}

The input data used as constant terms are called constant parameters. In the proposed design method, several calculations have to be done prior to the optimization itself.

It has been supposed that the cost of installing a traction substation depends mainly on the place where it has been located. After studying the railways to be electrified and the possible connections to the available three-phase networks, the railway has to be partitioned into zones and the best feeding solution has to be identified for each zone. For each zone, the following parameters have to be specified:

- $\quad$ Position where each zone ends.

- Cost of installing a substation in each zone. Both the cost of the substation and the cost of the line to connect it to the selected three-phase network are included.

- $\quad$ Maximum power that could be delivered by a substation depending of the zone it was installed. This value has to be established to avoid (i) excessive voltage drop or excessive unbalances in the three-phase network, and (ii) excessive power flow through the transformers of the substation.

To perform this optimization, it has been assumed that a reduced set of catenary types (with associated autotransformers in dual systems) has previously been selected. A multi-criteria optimization can be done to determine this set [2], but actually it is not necessary for the purposes of this paper.

For each catenary type, $c$, the following parameters have to be specified:

- $\quad$ Impedance per length unit. In dual systems, the equivalent model [1] has to be used to represent them as simple systems.

- $\quad$ The impedance per length unit (see [1]) due to the autotransformers separation (for simple systems its value is 0 ). This parameter depends on the catenary line parameters.

- $\quad$ The cost per length unit of installing each catenary type.

- The cost of installing each catenary type in a subsector that does not depend on the length of catenary installed. It corresponds to the cost of the autotransformers and thus is 0 for simple systems.

Several operation scenarios are used to evaluate technical constraints. A scenario is a list of trains that are simultaneously consuming electrical power. To define each scenario, $s c$, the following parameters have to be specified for each train, $t$ :

- $\quad$ Position of each train in the railway.

- $\quad$ Power that consumes each train: $S_{t}$. 
Other parameters to be defined are:

- $\quad$ Length of the railways to be electrified

- Maximum acceptable voltage drop

- Impedance of substations (all the substations are assumed to have the same value).

- The current $I_{t}$ consumed by each train. It is estimated assuming an average voltage: $\bar{V}$

$$
I_{t}(s c, t)=\frac{S_{t}(s c, t)}{\bar{V}}
$$

\subsection{Variables}

The variables are quantities that are changed in order to minimize the total cost of construction and to fulfill with technical constraints. The variables defined in the proposed model are:

- Number of traction substations supplying the stretch considered for the optimization.

- Location of each substation along the stretch.

- Type of catenary used in each subsector.

\subsection{Objective function}

The total cost of construction of the electrification to be minimized (see Eq. (2)) includes the cost of building the substations, $C_{S u b}$, the cost of the catenaries, $C_{C a t}$, and the cost of the autotransformers, $C_{A T}$, (if dual systems are used).

$$
C=C_{S u b}+C_{C a t}+C_{A T}
$$

\subsection{Restrictions}

The following restrictions have been included in the genetic algorithm formulation as penalty factors added to the fitness function.

- $\quad$ Maximum acceptable voltage drop for the operating conditions.

- Maximum power that can be delivered by the substations.

From the genetic algorithm point of view, the dynamic behavior of these restrictions is remarkable. That is, the restrictions are included in a penalty function that: is active in case of not fulfilling each restriction; and proportionally increases with the number of generations.

\subsection{Genetic algorithm}

Over the years, much work has been done in engineering optimization and the current tendency is to deal with real-life applications which are multi-objective by nature. Therefore, a standard genetic algorithm has been applied to the 
computation of the aforementioned optimization problem, although some specific tools and functions have been tailored too. Thus, its main characteristics can be summarized as follows:

- The individuals have been codified in binary code, including each chromosome the optimization variables described in section 3.2.

- The mechanisms of nature used for this optimization have been: a Roulette-based algorithm for the selection process; and monopoint schemes for crossover and mutation processes, whose parameters of probability are collected in Table 1 .

- $\quad$ The fitness function that leads the genetic algorithm has been build by the objective function, section 3.3, and the penalty functions derived from section 3.4, as follows:

$$
\begin{gathered}
F=C_{S u b}+C_{C a t}+C_{A T}+\pi \\
\pi=\pi_{d} \cdot \pi_{s} \\
\pi_{d}=(1+\beta \cdot(n-1)) \\
\pi_{s}=r \cdot v
\end{gathered}
$$

where $F$ is the fitness function which depends of the different costs involved in optimization problem and a penalty factor. This penalty function is composed of an static and a dynamic part as can be seen in equation (4), where: $r$ is the static penalty, $\beta$ is the dynamic penalty, $n$ is the number of generations computed and $v$ the degree of violation of each restriction.

Table 1 collects the numeric parameters of the genetic algorithm used in the numerical simulation correspond to standard values of randomized operators and another related to penalty functions.

Table 1: $\quad$ Genetic algorithm parameters.

\begin{tabular}{lc}
\hline \multicolumn{1}{c}{ Property } & Value \\
\hline Static penalty, $r$ & $10^{6}$ \\
Dynamic penalty $\beta$ & 0.2 \\
Crossover probability & 0.7 \\
Mutation probability & 0.03 \\
Population size & 200 \\
\hline
\end{tabular}

\section{Example}

The proposed model has been used to obtain the power supply of a section of the new Madrid - Barcelona high-speed line between the km 306 and km 441.6.

The scenarios that have been considered in this study case correspond to traffic meshes of trains consuming 24MVA (typically double compositions of 12MVA trains) separated by $24 \mathrm{~km}$. These hypotheses correspond to high-speed trains circulating at $350 \mathrm{~km} / \mathrm{h}$, with frequencies of $4 \mathrm{~min}$.

Table 2 shows the considered catenary repository. It has been obtained by using the multi-criteria optimization procedure described in [3]. 
Table 2: $\quad$ Catenary repository to be used.

\begin{tabular}{|c|c|c|c|c|c|}
\hline Catenary & C5 & C4 & C3 & C2 & C1 \\
\hline Contact wire & Bz 150mm2 & $\begin{array}{c}\mathrm{Bz} \\
150 \mathrm{~mm} 2\end{array}$ & $\begin{array}{c}\mathrm{Bz} \\
150 \mathrm{~mm} 2\end{array}$ & $\begin{array}{c}\mathrm{Bz} \\
150 \mathrm{~mm} 2\end{array}$ & $\begin{array}{c}\mathrm{Bz} \\
150 \mathrm{~mm} 2\end{array}$ \\
\hline Sustainer wire & Bz 100mm2 & $\begin{array}{c}\mathrm{Bz} \\
100 \mathrm{~mm} 2\end{array}$ & $\begin{array}{c}\mathrm{Bz} \\
100 \mathrm{~mm} 2\end{array}$ & $\begin{array}{c}\mathrm{Bz} \\
100 \mathrm{~mm} 2\end{array}$ & $\begin{array}{c}\mathrm{Bz} \\
100 \mathrm{~mm} 2\end{array}$ \\
\hline Positive feeder & Without feeder & $\begin{array}{c}\text { Without } \\
\text { feeder }\end{array}$ & LA-110 & LA-280 & LA-280 \\
\hline Negative feeder & LA-110 & LA-380 & LA-280 & LA-380 & LA-380 \\
\hline Return wire & LA-110 & LA-280 & LA-110 & LA-280 & LA-280 \\
\hline Number of AT per sector & 1 & 1 & 1 & 1 & 2 \\
\hline ZCAT (ohm/km) & 0,100530 & 0,085893 & 0,072287 & 0,066348 & 0,066348 \\
\hline CFIX (units) & 200 & 200 & 200 & 200 & 400 \\
\hline CCAT (units/km) & 26,136 & 28,140 & 30,900 & 33,840 & 33,840 \\
\hline ZDESV (ohm $/ \mathrm{km})$ & 0,037155 & 0,036300 & 0,033548 & 0,028995 & 0,014497 \\
\hline
\end{tabular}

Table 3 shows the cost of installing a substation and the maximum power that could be delivered depending on the location.

Table 3: $\quad$ Zone partitioning for installing a substation.

\begin{tabular}{|c|c|c|c|c|}
\hline From km & To km & CZONE & PZONE & Description \\
\hline 306 (Start) & 341,5 & 1000 & $60 \mathrm{MVA}$ & Fed from 220kV network \\
\hline 341,5 & 346 & $\infty$ & - & $\begin{array}{c}\text { Forbidden due to environmental } \\
\text { protection }\end{array}$ \\
\hline 346 & 350 & 1500 & $100 \mathrm{MVA}$ & Fed from 220kV network \\
\hline 350 & 377 & $\infty$ & - & $\begin{array}{c}\text { Forbidden due to environmental } \\
\text { protection }\end{array}$ \\
\hline 377 & 397 & 2500 & $100 \mathrm{MVA}$ & Fed from 400kV network \\
\hline 397 & 410 & $\infty$ & - & $\begin{array}{c}\text { Forbidden due to environmental } \\
\text { protection }\end{array}$ \\
\hline 410 & 413 & 2000 & $60 \mathrm{MVA}$ & Fed from 220kV network \\
\hline 413 & 415 & $\infty$ & - & $\begin{array}{c}\text { Forbidden due to environmental } \\
\text { protection }\end{array}$ \\
\hline 415 & 441.6 (End) & 2000 & $60 \mathrm{MVA}$ & Fed from 220kV network \\
\hline
\end{tabular}

The results obtained can be discussed in two main aspects: on the one hand the robustness of the genetic algorithm and; on the other hand, the accuracy by comparing with another optimization procedure.

Figure 4 shows the fitness function evolution along generations of the genetic algorithm. In different colors are plotted: the optimum evolution, which is reached with only 11 generations; and the first and second quartiles, which can give an idea of the robust behavior of the algorithm. It seems clear that at the tenth generation penalized individuals disappear of most of the solutions. Thus, at least the $50 \%$ of possible solutions are feasible and their fitness values are exactly the costs of the objective function.

Figure 5 shows the location of substations and subsectors; and the types of catenaries (red lines refers to the solution obtained with the genetic algorithm and blue lines to the MIP-based one). Marked with circles are pointed out the location of substations along the stretch considered, between the $\mathrm{km} 306$ and $\mathrm{km}$ 441.6 of the Madrid-Barcelona high speed line. However, the horizontal axis 
shows the relative kilometer points, from $\mathrm{km} 0$ to $\mathrm{km} 135.6$, to make clearer the understanding. Moreover, the vertical axis means the type of catenary used in each subsector. As well as in Figure 5, Table 4 collects the numeric catenary assignment that has been obtained with the GA optimization, while Table 5 shows the assignment obtained with the MIP optimization.

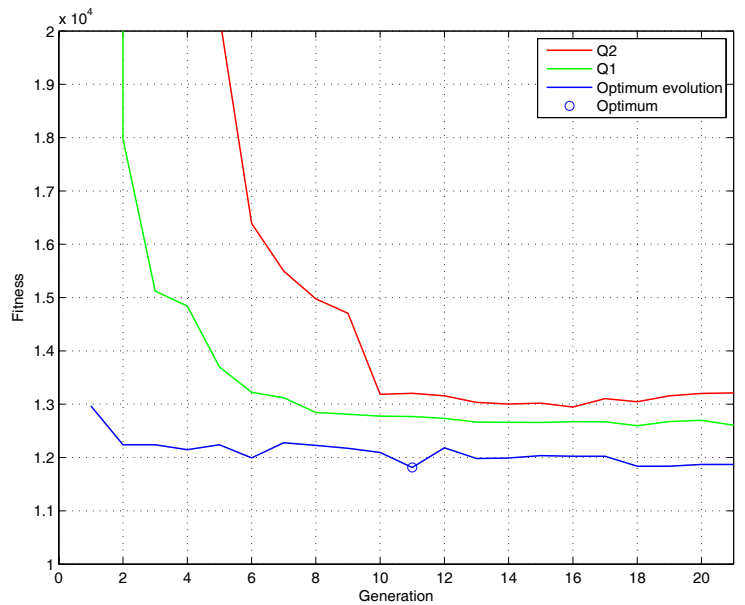

Figure 4: Fitness evolution.

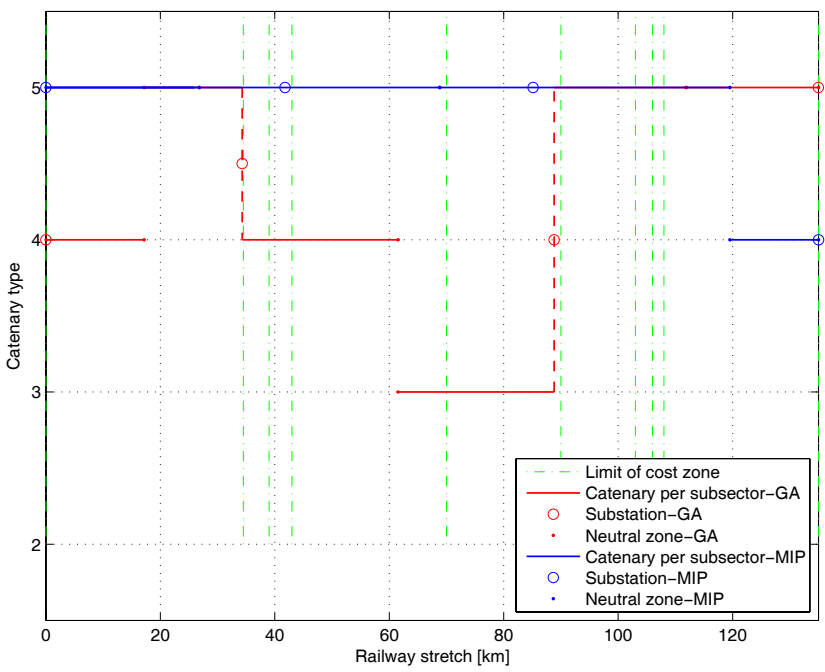

Figure 5: Location of substations and subsectors; and types of catenaries.

Table 6 compares the minimum total cost of the electrification obtained with both methods as well their computation time. The proposed genetic algorithm finds similar solutions in terms of total cost in significantly shorter time. 
Table 4: $\quad$ Catenary assigned to each sector in GA Model.

\begin{tabular}{|l|l|c|}
\hline \multicolumn{1}{|c|}{ From km } & \multicolumn{1}{c|}{ To km } & Catenary \\
\hline 306.000 (substation) & 323.15 (neutral zone) & C4 \\
\hline 323.15 (neutral zone) & 340.3 (substation) & C5 \\
\hline 340.3 (substation) & 367.55 (neutral zone) & C4 \\
\hline 367.55 (neutral zone) & 394.8 (substation) & C3 \\
\hline 394.8 (substation) & 417.9 (neutral zone) & C5 \\
\hline 417.9 (neutral zone) & 441 (substation) & C5 \\
\hline
\end{tabular}

Table 5: $\quad$ Catenary assigned to each sector in MIP Model.

\begin{tabular}{|l|l|c|}
\hline \multicolumn{1}{|c|}{ From km } & \multicolumn{1}{c|}{ To km } & Catenary \\
\hline 306.000 (substation) & 332.791 (neutral zone) & C5 \\
\hline 332.791 (neutral zone) & 347.791 (substation) & C5 \\
\hline 347.791 (substation) & 374.824 (neutral zone) & C5 \\
\hline 374.824 (neutral zone) & 391.138 (substation) & C5 \\
\hline 391.138 (substation) & 425.534 (neutral zone) & C5 \\
\hline 425.534 (neutral zone) & 441.200 (substation) & C4 \\
\hline
\end{tabular}

Table 6: Compared results between MIP and GA models.

\begin{tabular}{|c|c|c|}
\hline Model & Global Cost & CPU Time \\
\hline GA & $11813 €$ & $1: 05: 42$ Pentium- $4,1.7 \mathrm{GHz}$ \\
\hline MIP & $11760 €$ & $0: 00: 58$ Pentium- $4,1.7 \mathrm{GHz}$ \\
\hline
\end{tabular}

\section{Conclusions}

This paper has presented a genetic algorithm based procedure to minimize the total cost of construction of the power supply while satisfying technical constraints such as voltage drops or power limitations.

To evaluate the performance of the model, a section of the new Madrid Barcelona high-speed line has been studied with a MIP optimization and with the proposed genetic algorithm optimization. Both procedures have found a similar solution in terms of costs, but the proposed algorithm performs the optimization in a significantly shorter time.

As the genetic-algorithm approach has obtained very promising results, future developments will be focused on designing particular evolutionary strategies to enhance the convergence. Additionally, more complex technical restrictions will be considered.

\section{References}

[1] E. Pilo, L. Rouco, and A. Fernández, "A reduced representation of $2 \times 25 \mathrm{kV}$ electrical systems for high-speed railways.," presented at IEEE/ASME Joint Rail Conference, Chicago, 2003. 
[2] E. Pilo, L. Rouco, and A. Fernández, "Catenary and autotransformer coupled optimization for $2 \times 25 \mathrm{kV}$ systems planning," WIT Transactions On the Built Environment, vol. 78, pp. pp.747-756, 2006.

[3] E. Pilo, L. Rouco, A. Fernández, "An optimization procedure to determine the topology of ac railways power supply networks," 2007 ASME/IEEE Joint Rail Conference \& Internal Combustion Engine Spring Technical Conference (JRCICE2007). Pueblo, Colorado, USA, March 13-16, 2007

[4] J. D. Glover, A. Kusko, and S. M. Peeran, "Train voltage analysis for AC railroad electrification," IEEE Transactions on Industry Applications, vol. IA-20, pp. 925-934, 1984.

[5] C. Gourdon and C. Herce, "The overhead system of the TGV-Atlantique," presented at International Conference on Main Line Railway Electrification (Conf. Publ. no.312), London, UK, 1989.

[6] H. Roussel, "Power supply for the Atlantic TGV high-speed line," presented at IEE International Conference on Main Railway Electrification, York, 1989.

[7] R. J. Hill and I. H. Cevik, "Parallel computer simulation of autotransformerfed AC traction networks," presented at ASME/IEEE Joint Railways Conference, 1990. 